BMJ Open

Diabetes

Research

\& Care

\title{
Addition of a single short-acting insulin bolus to basal insulin-supported oral therapy: a systematic review of data on the basal-plus regimen
}

\author{
Jochen Seufert, ${ }^{1}$ Anja Borck, ${ }^{2}$ Peter Bramlage (i) ${ }^{3}$
}

To cite: Seufert J, Borck A, Bramlage P. Addition of a single short-acting insulin bolus to basal insulin-supported oral therapy: a systematic review of data on the basal-plus regimen. BMJ Open Diab Res Care 2019;7:e000679. doi:10.1136/ bmjdrc-2019-000679

Received 3 April 2019 Revised 28 June 2019 Accepted 12 September 2019

Check for updates

(C) Author(s) (or their employer(s)) 2019. Re-use permitted under CC BY-NC. No commercial re-use. See rights and permissions. Published by BMJ.

${ }^{1}$ Division of Endocrinology and Diabetoligy, Department of Medicine II, Medical Center University of Freiburg, Faculty of Medicine, University of Freiburg, Freiburg, Germany ${ }^{2}$ Sanofi, Berlin, Germany ${ }^{3}$ Institute for Pharmacology and Preventive Medicine, Cloppenburg, Germany

Correspondence to Professor Jochen Seufert: jochenseufert@uniklinikfreiburg.de

\section{ABSTRACT}

We summarize here clinical and trial data on a once-daily administration of a single bolus to the meal with the largest expected postprandial glucose excursion (basalplus), and comment on its clinical utility in the treatment of type 2 diabetes. A PubMed search of data published until September 2018 was taken into consideration and PRISMA (Preferred Reporting Items for Systematic Reviews and Meta-Analyses) guidelines were followed. Eighteen reports representing 15 studies were identified (age: 18-80 years; 50-890 patients; follow-up: 8 days to 60 weeks). Data suggest basal-plus is efficacious for improving glycemic control, with a low incidence of (severe) hypoglycemia and minor increases in bodyweight. The timing of short-acting insulin administration and use of different monitoring/ titration approaches appear to have minimal impact. When compared with premixed insulin, basal-plus results in largely comparable outcomes. Compared with basalbolus, it may result in non-inferior glycemic improvements with less weight gain, less hypoglycemia and fewer daily injections. A basal insulin/glucagon-like peptide-1 receptor agonist fixed ratio combination may offer several advantages over the basal-plus regimen, at the cost of gastrointestinal side effects. We conclude that the stepwise introduction of short-acting insulin via the basal-plus strategy represents a viable alternative to a full basal-bolus regimen and may help to overcome barriers associated with multiple injections and anticipated complexity of the insulin regimen.

\section{INTRODUCTION}

Type 2 diabetes mellitus (T2DM) is characterized by progressive deterioration of endogenous insulin secretion and subsequently impaired glycemic control. As a consequence, stepwise intensification of individualized treatment strategies are necessary to achieve normoglycemia in the patient. This commonly involves starting with diet and exercise regimens, and progressing through oral antidiabetic drug (OAD) treatment to eventual supplementation with insulin. ${ }^{1}$ The combination of one or more OADs and basal insulin is commonly denoted as basal (insulin)-supported oral therapy (BOT). Near-normal fasting blood glucose (FBG) values are generally achieved and maintained by BOT, thanks to its primary effect on overnight fasting glucose; however, prandial glucose excursions may remain inadequately controlled by BOT contributing to the overall glucose burden and level of glycosylated hemoglobin (HbAlc) over time. ${ }^{2}$ One approach to restoring satisfactory postprandial glycemic control is the addition of prandial short-acting insulin to the basal regimen mimicking physiological patterns of endogenous insulin secretion.

Due to concerns regarding weight gain, hypoglycemia, injection burden and impact on daily quality of life, both physicians and patients are frequently reluctant to implement insulin intensification by the abrupt addition of multiple daily prandial insulin boluses to existing BOT (basal-bolus). ${ }^{3}{ }^{4}$ This reluctance may result in clinical inertia, poor adherence and/or poor persistence. ${ }^{5}$ One alternative is to switch from BOT to twice-daily premixed insulin, which provides both basal and prandial coverage due to its biphasic pharmacokinetic properties. ${ }^{6}$ Although this addresses several of the aforementioned issues, flexibility is limited by the fixed ratio of long-acting to short-acting components, precluding self-titration of prandial doses based on self-monitored blood glucose (SMBG). ${ }^{7}$ A so-called basalplus approach has gained interest, in which BOT is intensified by addition of a once-daily short-acting insulin bolus only, typically prior to the main meal of the day. The rationale behind this simple approach is that it blunts the most significant postprandial glucose peak, thereby slowing disease progression while preserving dose flexibility, limiting the risk of hypoglycemia and weight gain and 


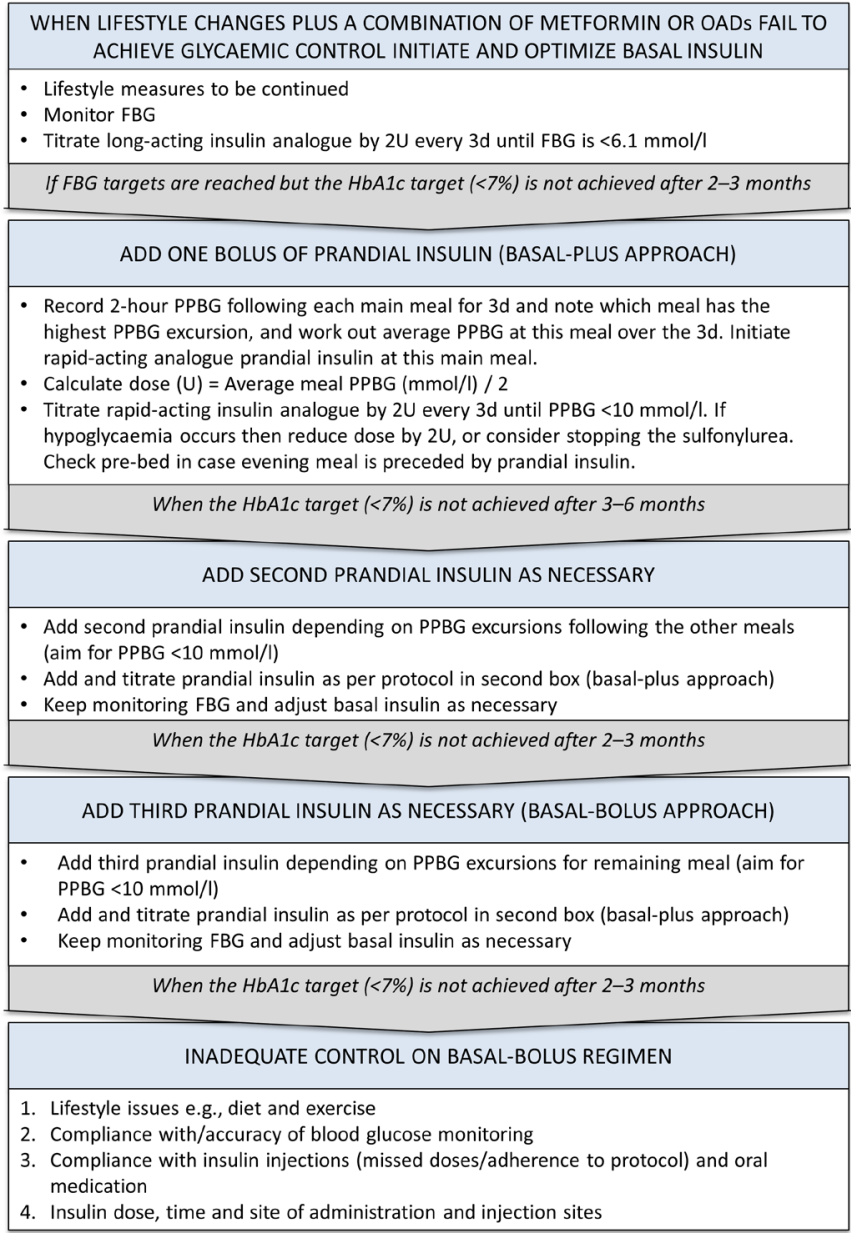

Figure 1 Suggested algorithm for the stepwise initiation and titration of rapid-acting insulin (adapted from Owens et al). ${ }^{10} \mathrm{D}$, day; FBG, fasting blood glucose; HbA1c, glycosylated hemoglobin; OAD, oral antidiabetic drug; PPBG, postprandial blood glucose; $U$, international units of insulin.

restricting the number of daily insulin injections to two. ${ }^{8}$ This approach may make intensification more acceptable to patients, and leave room for the stepwise addition of more prandial boluses in case glycemic targets are not met. ${ }^{9}$ A suggested protocol for this stepwise approach can be found in figure $1 .^{10}$

In recent years, a number of studies have been published that compare the basal-plus regimen to alternative strategies, or explore different aspects of its clinical implementation. We aimed to gather available evidence in a systematic review of the literature and consolidate our findings into a comprehensive overview from which to derive clinical recommendations.

\section{METHODS}

The present systematic review (no predefined protocol) of published full-text articles is based on the PRISMA (Preferred Reporting Items for Systematic Reviews and Meta-Analyses) approach for evaluating healthcare interventions. ${ }^{11} \mathrm{~A}$ schematic of the steps taken to identify the included studies is shown in figure 2. Briefly, on

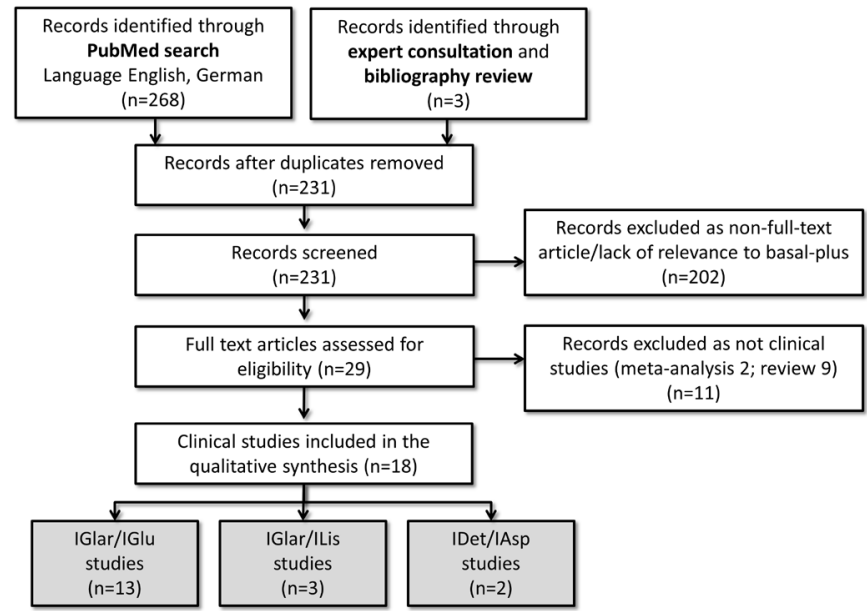

Figure 2 PRISMA flow diagram of clinical study selection. IAsp, insulin aspart; IDet, insulin detemir; IGlar, insulin glargine; IGlu, insulin glulisine; ILis, insulin lispro; PRISMA, Preferred Reporting Items for Systematic Reviews and MetaAnalyses.

September 22 2018, studies relevant to the basal-bolus regimen were identified through a series of PubMed searches, scanning reference lists of retrieved articles, and consultation with experts in the field. Only English and German language articles were considered, with no further eligibility criteria specified. A total of three PubMed searches were carried out using the following search terms:

1. "("insulin"[MeSH Terms] OR "insulin"[All Fields]) AND ("diabetes mellitus"[MeSH Terms] OR ("diabetes"[All Fields] AND "mellitus"[All Fields]) AND basal[All Fields] AND prandial[All Fields]) AND Clinical Study[ptyp]" with no further limits applied (search 1); yielding 152 records 2. "basal-plus[All Fields]" with no further limits applied (search 2); yielding 102 records

3. "( "insulin" [MeSH Terms] OR "insulin"[All Fields]) AND ("diabetes mellitus"[MeSH Terms] OR ("diabetes"[All Fields] AND "mellitus"[All Fields]) OR "diabetes mellitus"[All Fields] OR "diabetes"[All Fields]) AND basal-plus[All Fields]) AND Review[ptyp]" with no further limits applied (search 3); yielding 14 records.

In addition, a bibliography review and consultation with experts in the field was carried out to identify any further pertinent studies (search 4). After removing duplicates, the titles and abstracts of the retrieved literature were screened, and those considered to be lacking relevance excluded. Full-text files for the remaining publications were obtained. The shortlisted articles were then sorted by type: review, meta-analysis or clinical study, with the latter further stratified according to the insulin combination used in the basal-plus group. Also, studies were labeled according to the treatment setting (inpatient vs outpatient) and are discussed separately.

Glucagon-like peptide-1 receptor agonists (GLP-1 RA) are increasingly used as combination partners for insulin. Especially, addition of a short-acting GLP-1 RA to basal insulin within a BOT regimen may represent a valid bona 
fide treatment intensification strategy. Therefore, we identified and included a single trial with lixisenatide as comparator into this systematic review. The results of the GLP-1 RA study are presented and discussed systematically together with the insulin trials.

Data from the clinical studies regarding study design, inclusion criteria, primary endpoints, and key outcomes were extracted into table 1, according to the PICO scheme. ${ }^{12}$ Details of intervention and comparator were recorded. Where applicable, implementation strategies for the basal-plus regimen were noted and are presented together with the outcomes. Further, data on HbA1c, FBG, bodyweight and hypoglycemia were extracted into table 2. Change in $\mathrm{HbA1c}$ from baseline and the proportion of patients achieving HbAlc $<7 \%$ were considered the principal summary measures. Whenever possible, data not available from the respective publications were retrieved from study investigators. No further statistical analyses were performed.

\section{RESULTS}

\section{Study characteristics}

The majority of clinical studies were randomized controlled trials (RCTs) comparing two or more treatment strategies (table 1). In addition, data from one retrospective chart analysis ${ }^{13}$ and one post hoc analysis of an RCT that was considered to provide additional value to the main analysis were extracted. ${ }^{14}$ All studies contained a basal-plus arm and included adult patients with T2DM only (age range, 18-80 years). In almost all cases, patients were required to be receiving insulin (commonly in the form of BOT) at the point of inclusion, with only a few studies stating prior OAD use as a minimum prerequisite. ${ }^{9}{ }^{1516}$ Study populations ranged from 50 to 890 patients, with the shortest follow-up lasting just 8 days and the longest 60 weeks. The basalplus regimen was composed of insulin glargine $100 \mathrm{U} /$ $\mathrm{mL}$ (IGlar) and insulin glulisine (IGlu) in 11 studies (13/18 manuscripts), ${ }^{8} 913$ 15-24 IGlar and insulin lispro (ILis) in 2 studies (3/18 manuscripts) ${ }^{142526}$ and insulin detemir (IDet) and insulin aspart (IAsp) in 2 studies $\left(2 / 18\right.$ manuscripts) ${ }^{27} 28$

\section{Glycosylated hemoglobin}

The change in HbA1c between baseline and final follow-up and the proportions of patients achieving HbAlc $<7 \%$ were common outcomes of the included studies. For IGlar/IGlu basal-plus regimens, the reduction in HbA1c was found to be comparable regardless of whether IGlu was administered before breakfast (baseline 7.35 \pm 0.71 ; reduction $-0.31 \%$ ) or before the main meal (baseline $7.29 \pm 0.69$; reduction $-0.36 \%$; $\mathrm{p}<0.0001$ for equivalence $)^{8}$ (table 2). However, the proportion of patients with prior HbAlc values $>7 \%$ achieving a final value $<7 \%$ was significantly higher for the main meal group $(52.2 \%$ vs $36.5 \%$; $\mathrm{p}=0.028$ ). For IGlar/ILis combinations, a subanalysis of a trial reported by Tinahones et al found main meal timing to make very little difference to HbAlc reductions. ${ }^{14}$ Telemonitoring and SMBG approaches also resulted in comparable changes in $\mathrm{HbAlc}$ and the proportion of IGlar/IGlu patients achieving the $<7 \%$ target. $^{15}$ In terms of IDet/IAsp combinations, the HbAlc values of basal-plus patients improved significantly, regardless of the assessment regimen (preprandial or postprandial glucose) used to determine intensification needs. ${ }^{28}$

An IGlar/IGlu basal-plus regimen was shown to be superior to BOT for reducing HbAlc $(-0.37 \%$ vs $-0.11 \%$; $\mathrm{p}=0.0290)$ and facilitating target attainment $(22.4 \%$ vs $8.8 \%$ of patients; $\mathrm{p}<0.05) .{ }^{21}$ For both of these parameters, all IGlar/IGlu studies found basal-plus to be non-inferior to premix insulin regimens (All To Target trial: $-2.1 \%$ vs $-1.8 \%$ and $49 \%$ vs $39 \%$; Jin et al: $-0.9 \%$ vs $-1.0 \%$ and $33.3 \%$ vs $29.3 \%$; Vora et al: $-1.0 \%$ vs $-1.22 \%$ and $20.6 \%$ vs $27.9 \%$, respectively). ${ }^{1622} 23$ Conversely, twice-daily ILis low mixture (LM25) was found to result in a significantly greater reduction in HbAlc compared with an IGlar/ ILis basal-plus regimen (LS mean treatment difference: $-0.22 ; 95 \%$ CI: -0.39 to $-0.05 ; \mathrm{p}=0.010)$, though no significant difference was seen for HbAlc target achievement $(34.5 \%$ vs $30.0 \%) .{ }^{26}$ Lixisenatide was shown to be comparable to basal-plus for $\mathrm{HbAlc}$ reduction (treatment difference: $-0.1,95 \%$ CI: -0.17 to 0.06 ) and HbAlc $<7 \%$ (treatment difference: $3.7,95 \%$ CI: -4.03 to 11.49 ). ${ }^{24}$

Davidson et alshowed an IGlar/IGlu basal-plus regimen to be non-inferior to basal-bolus in terms of reducing HbAlc $(-0.44 \%$ vs $-0.43 \%) .{ }^{9}$ However, non-inferiority was not demonstrated by the OSIRIS study $(-0.40 \%$ vs $-0.72 \%) .{ }^{20}$ Moreover, fewer patients in both studies met the $<7 \%$ target on the basal-plus regimen (Davidson et al: $30 \%$ vs $46 \%, p=0.017$; OSIRIS: $18.4 \%$ vs $27.1 \%$; $95 \%$ CI for treatment difference: -17.92 to 0.48$).{ }^{9}{ }^{20}$ Similarly, while an IDet/IAsp study found the basal-plus and basalbolus regimens to result in statistically similar reductions in $\mathrm{HbAlc}(-0.98 \%$ vs $-1.12 \%)$ and proportions of patients meeting the HbAlc target (55.9\% vs $63.3 \%$ ), a similar trend toward better outcomes with basal-bolus was apparent. ${ }^{27}$

Given the short timeframe of inpatient studies, improvement in daily blood glucose (rather than HbAlc) was measured. This was found to be similar between hospitalized patients on premixed insulin+ILis and IGlar/ILis basal-plus regimens $(-100.7 \mathrm{vs}-70.7 \mathrm{mg} / \mathrm{dL}$ by the end of treatment; $p=0.107),{ }^{25}$ and between IGlar/IGlu basalbolus and basal-plus regimens (approximately $-44 \mathrm{mg}$ / $\mathrm{dL}$ after treatment day 1 in both cases). ${ }^{18}$

\section{Fasting blood glucose}

FBG values at baseline were extremely heterogeneous between studies, ranging from 103.9 to $198.2 \mathrm{mg} / \mathrm{dL}$ for basal-plus arms (table 2). The lowest of these values were reported by trials including patients who were already taking basal insulin at baseline, ${ }^{8} 132224$ 26-28 which also reported only small changes in FBG over the course of the study (maximum increase: $+13.51 \mathrm{mg} / \mathrm{dL}$ at 24 weeks; ${ }^{26}$ maximum decrease: $-16 \mathrm{mg} / \mathrm{dL}$ at 48 weeks $^{28}$ ). Only one 


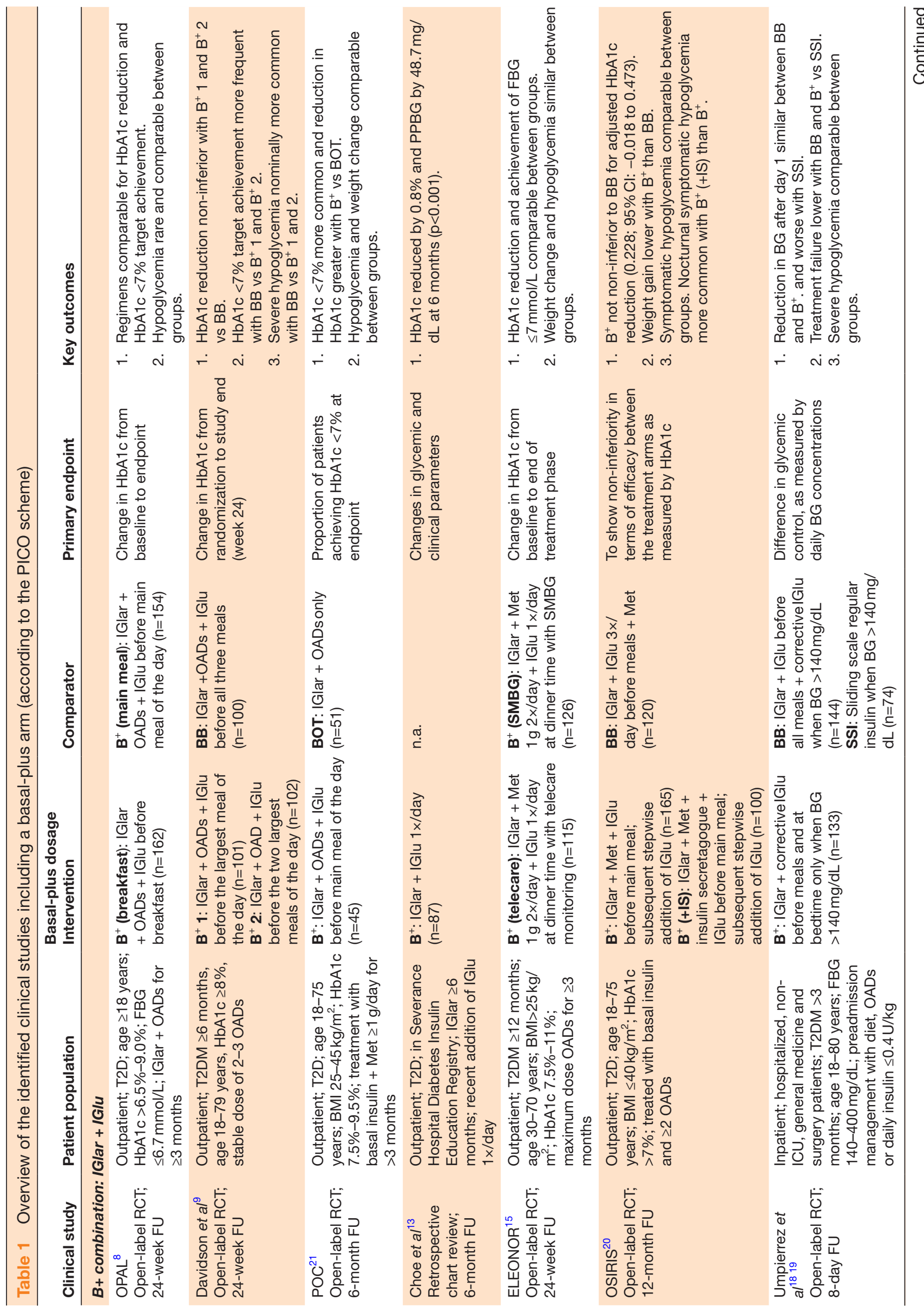




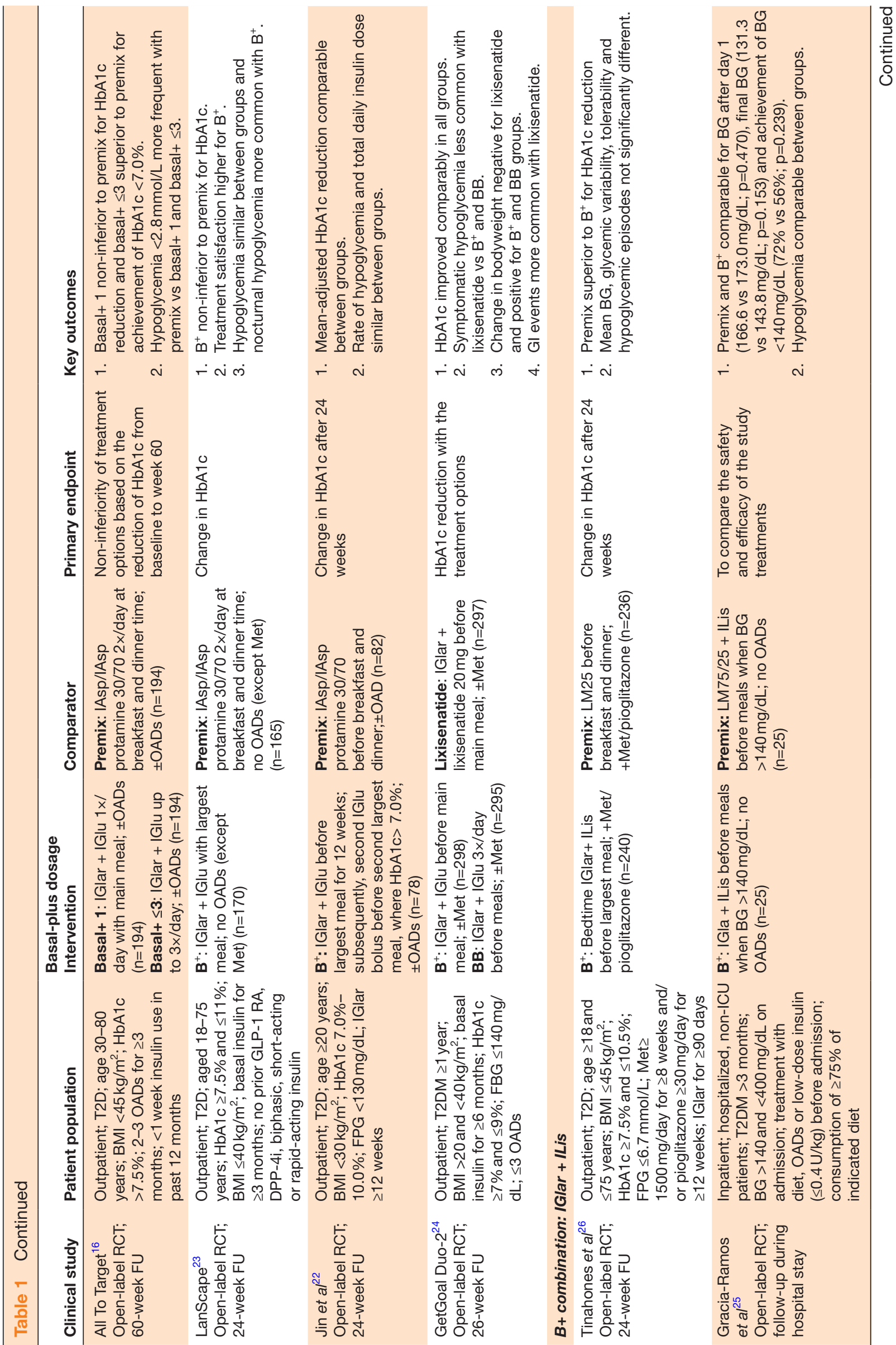




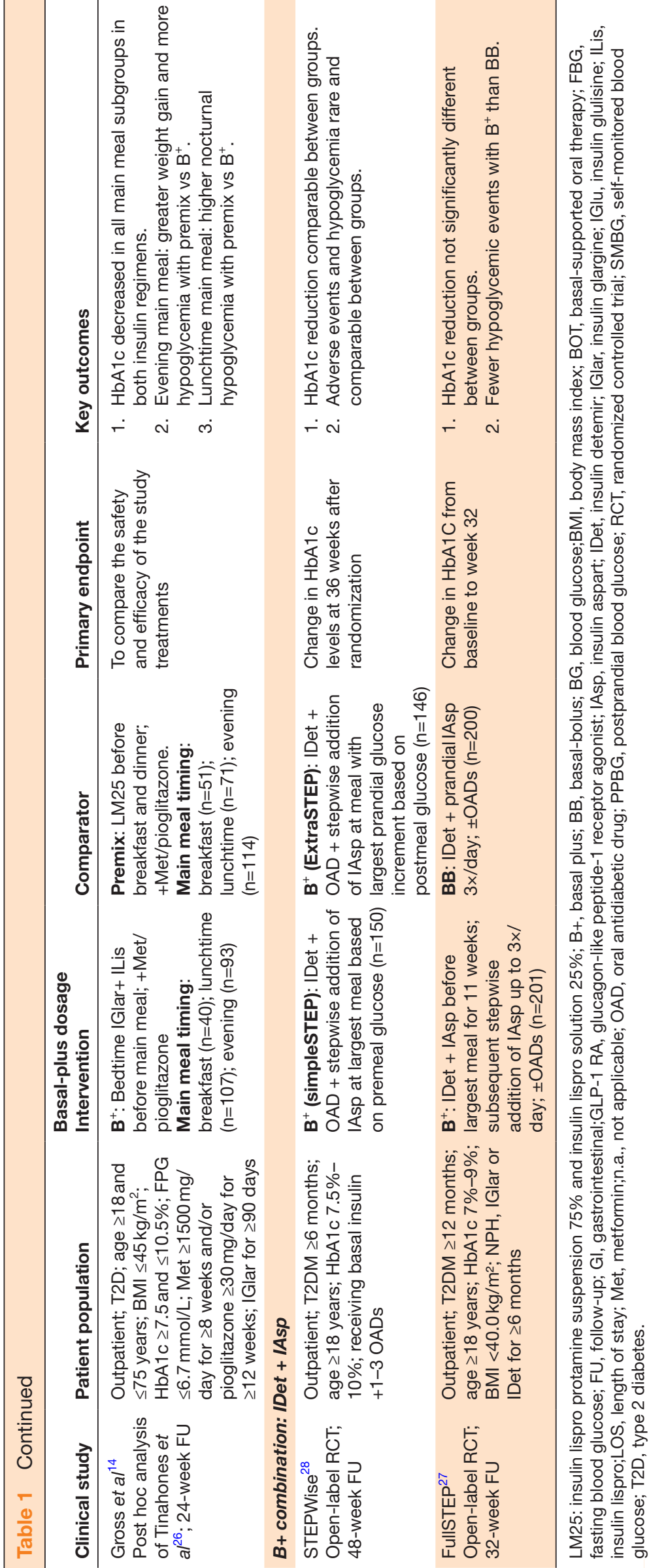




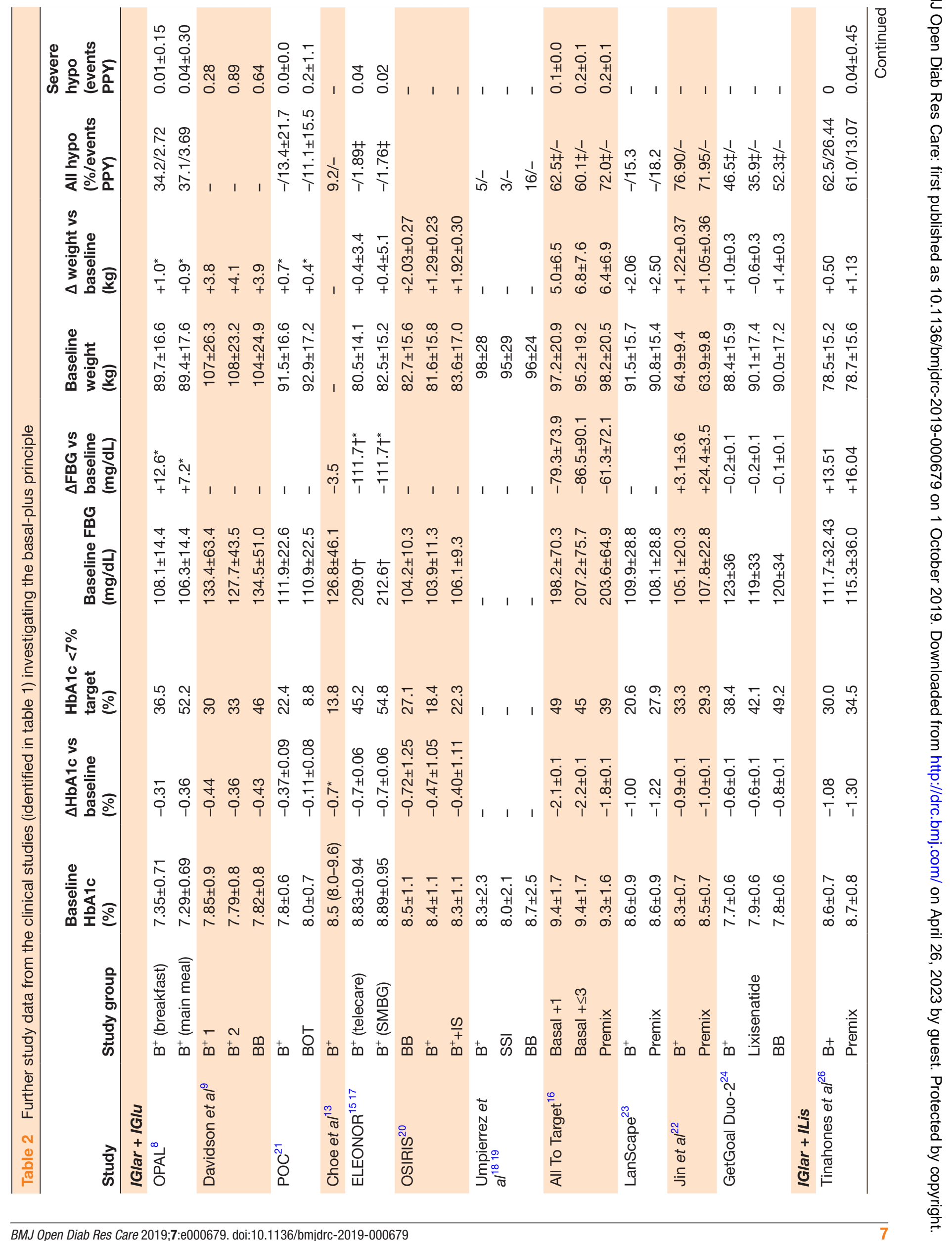




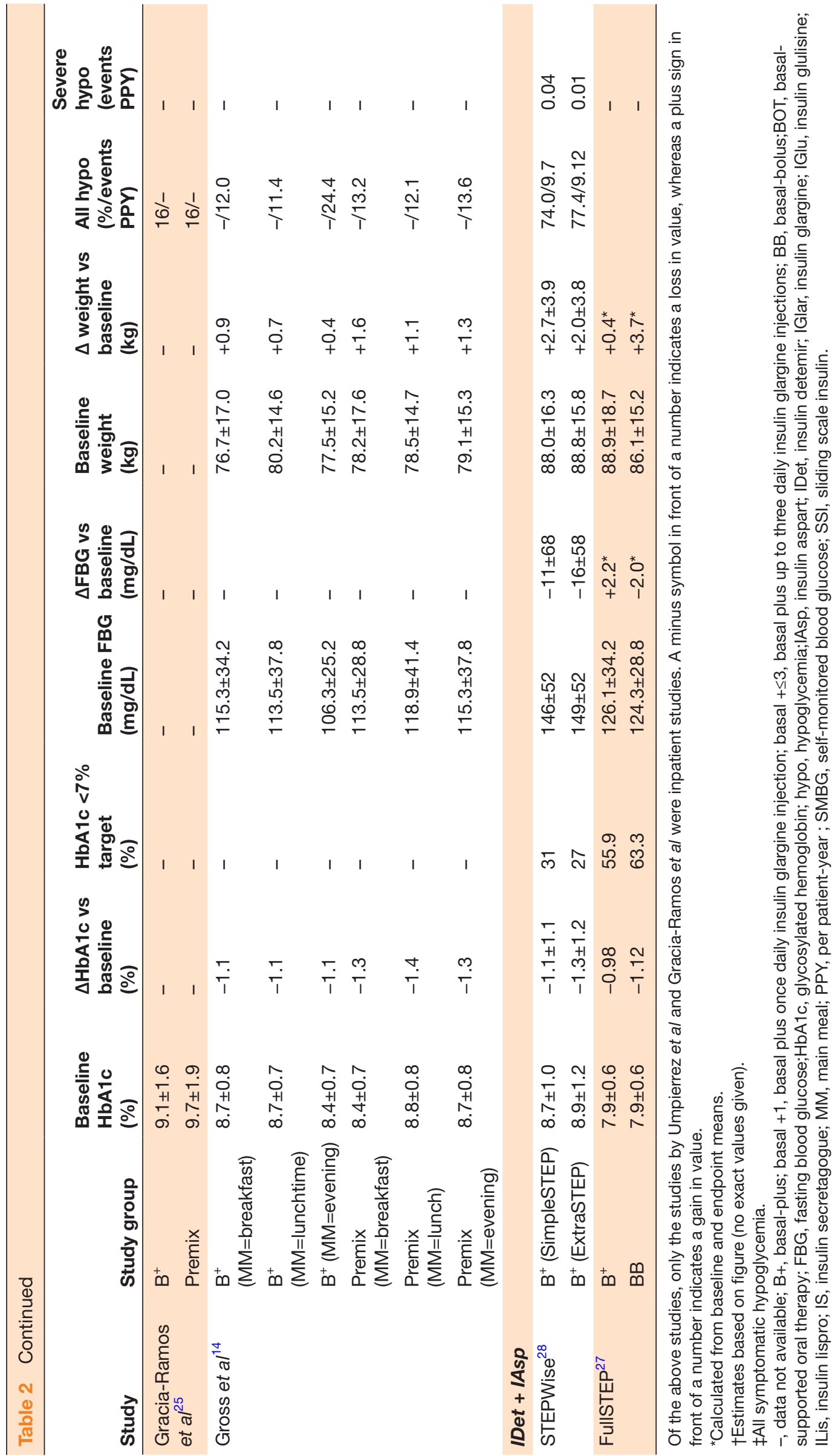


study showed FBG changes to be significantly different from a comparator regimen (premixed insulin: $+3.1 \mathrm{vs}$ $+24.4 \mathrm{mg} / \mathrm{dL} ; \mathrm{p}=0.0001^{22}$ ), and neither meal timing nor titration approach had a significant influence. ${ }^{1428}$

Baseline FBG values were highest in studies where patients were only required to be taking OADs at the time of inclusion. ${ }^{9} 1516$ The ELEONOR study reported the basal-plus approach to result in a significant fall in FBG (approximately $-111.7 \mathrm{mg} / \mathrm{dL}$ by week 20 ), regardless of the glucose monitoring approach employed (telecare vs SMBG).$^{15}$ Concurrently, a large reduction in FBG was reported for the basal-plus arm in the All To Target trial $(-79.3 \mathrm{mg} / \mathrm{dL})$, which was significantly greater than in the premixed insulin arm $(-61.3 \mathrm{mg} / \mathrm{dL})$, with the basal-bolus regimen resulting in the greatest decrease $(-86.5 \mathrm{mg} / \mathrm{dL}){ }^{16}$

\section{Bodyweight}

The effect of the basal-plus regimen on bodyweight was a secondary endpoint in the majority of studies. All these found patients to gain weight over the study period, though a wide range of magnitudes are reported $(+0.4-+5.0 \mathrm{~kg}) .{ }^{1516}$ Heterogeneity in terms of study duration, insulin combinations, prior insulin exposure and concomitant OAD medications are likely to be responsible for this difference.

For IGlar/IGlu combinations, administration of shortacting prandial insulin before breakfast as compared with the main meal appeared to make little difference to the change in bodyweight at 24 weeks $(+1.0 \mathrm{vs}+0.9 \mathrm{~kg}),{ }^{8}$ with the same outcome regarding use of telecare and SMBG monitoring approaches (both $+0.4 \mathrm{~kg})^{15}$ (table 2). In addition, the use of preprandial glucose levels compared with postprandial excursions to determine insulin titration did not result in a significant difference in weight gain by week $36(+2.7 \mathrm{vs}+2.0 \mathrm{~kg}){ }^{28}$

An IGlar/IGlu basal-plus regimen was shown to be statistically comparable to BOT $(+0.7 \mathrm{vs}+0.4 \mathrm{~kg}),{ }^{21}$ as well as premixed insulin (Jin et al: $+1.22 \mathrm{vs}+1.05 \mathrm{~kg}$; Riddle et al: $+5.0 \mathrm{vs}+6.4 \mathrm{~kg}$; Vora et al: $+2.06 \mathrm{vs}+2.50 \mathrm{~kg}) .{ }^{1622} 23$ For IGlar/ ILis combinations, patients on the basal-plus regimen were found to have gained significantly less weight compared with those on LM25 premixed insulin $(+0.5 \mathrm{vs}+1.13)$ after 24 weeks of treatment, ${ }^{26}$ with those administering IGlu before their main meal in the evening experiencing the smallest bodyweight increment $(+0.4 \mathrm{~kg}) .{ }^{14}$

IGlar/IGlu studies found weight gain to be significantly lower for basal-plus patients compared with basal-bolus patients at 1 year $(+1.29 \mathrm{vs}+2.03 \mathrm{~kg}),{ }^{20}$ and compared with those on up to three daily IGlu injections at 60 weeks $(+5.0$ vs $+6.8 \mathrm{~kg} ; \mathrm{p}=0.024) .{ }^{16}$ For IDet/IAsp combinations, the basal-plus regimen resulted in only a minimal weight increase at 32 weeks (approximately $+0.4 \mathrm{~kg}$ ), while basalbolus patients gained $\sim 3.7 \mathrm{~kg}$ (both values calculated from mean baseline and final weight in each group; no statistics provided). ${ }^{27}$ In contrast, Davidson et al reported no significant differences in the magnitude of bodyweight increment between patients receiving once, twice or thrice daily IGlu $(+3.8,+4.1$ and $+3.9 \mathrm{~kg}$, respectively) after 24 weeks. ${ }^{9}$ Patients taking lixisenatide were noted to have achieved weight loss at week $24(-0.6 \mathrm{~kg})$, while gains of +1.0 and $+1.4 \mathrm{~kg}$ were seen for basal-plus and basal-bolus groups, respectively. ${ }^{24}$ However, while lixisenatide treatment was shown to be statistically superior to the basal-bolus regimen $(\mathrm{p}<0.0001)$, the same was not true when compared with the basal-plus approach.

\section{Hypoglycemia}

Hypoglycemia occurred in basal-plus patients at an incidence of 1.76 to 26.44 events per patient-year (PPY) (table 2). It was unaffected by the use of telecare compared with SMBG monitoring approaches in basal-plus patients (1.89 vs 1.76 events PPY), ${ }^{15}$ by the use of premeal compared with postprandial excursion glycemic data to adapt titrations (9.7 vs 9.12 events PPY) ${ }^{28}$ and by administration of IGlu at breakfast time or before the main meal (2.72 vs 3.69 events PPY) ${ }^{8}$ However, administration of ILis with a main evening meal appeared to result in approximately double the incidence of hypoglycemia compared with administration with a main meal at breakfast or lunchtime (24.4 vs 12.0 and 11.4 events PPY, respectively). ${ }^{14}$

One study found the incidence of hypoglycemia PPY not to be statistically different between IGlar/IGlu and premixed insulin regimens (15.3 vs 18.2 events PPY; $\mathrm{p}=0.22),{ }^{23}$ with three other reporting comparable proportions of patients experiencing a hypoglycemic event during the study (Jin et al: $76.90 \%$ vs $71.95 \%$; Tinahones et al: $62.5 \%$ vs $61.0 \%$; Gracia-Ramos et al: $16 \%$ vs $16 \%) .{ }^{22} 2526$ Only one study found a basal-plus regimen to result in lower rates compared with premixed insulin $(62.5 \%$ vs $72.0 \%, \mathrm{p}<0.05){ }^{16}$

In the IGlar/IGlu OSIRIS trial, the incidence of hypoglycemia PPY was comparable between basal-plus and basal-bolus patients. ${ }^{20}$ Conversely, the FullSTEP study reported IDet/IAsp basal-plus patients to have significantly fewer hypoglycemic events overall as compared with basal-bolus patients (relative risk $(\mathrm{RR})=0.58$; $95 \%$ CI: 0.45 to $0.75 ; \mathrm{p}<0.0001) .{ }^{27}$ Similarly, the proportion of patients experiencing an event was reported to be significantly lower for hospitalized basal-plus compared with basal-bolus patients (Umpierrez et al: $5 \%$ vs $16 \%$, $\mathrm{p}=0.009) .{ }^{18}$ Lixisenatide resulted in lower proportions of patients experiencing hypoglycemia over 26 weeks $(35.9 \%$ vs $46.5 \% ; \mathrm{p}=0.01){ }^{24}$

Severe hypoglycemia was extremely rare $(<0.3$ events PPY) in all seven studies reporting this outcome. ${ }^{891516212628}$ However, only the trial with the highest hypoglycemia event rate reported significantly lower rates of severe hypoglycemia in the basal-plus group compared with its comparator, basal-bolus. ${ }^{9}$

\section{SUMMARY OF EVIDENCE: IMPLICATIONS FOR CLINICAL PRACTICE}

Overall, the above data suggest that the basal-plus regimen is efficacious for improving glycemic control 
in patients with T2DM, with a low incidence of hypoglycemia and generally minor increases in bodyweight. Based on a small body of available evidence, the timing of short-acting insulin administration and use of different monitoring/titration approaches appear to have minimal clinical impact on efficacy and safety. When compared with premixed insulin, the basal-plus approach appears to result in largely comparable outcomes, whereas compared with basal-bolus regimen, it may result in generally non-inferior glycemic improvements with less weight gain, less hypoglycemia and fewer daily injections. A basal insulin/lixisenatide combination may offer several advantages over the basal-plus regimen.

\section{Choice of patients for basal-plus}

In the investigated set of studies, ongoing use of a basal insulin \pm OADs accompanied by an elevated HbA1c level was a common prerequisite for inclusion. ${ }^{8} 131420-24$ 26-28 This explains the low baseline FBG levels and minimal changes in FBG seen in these particular studies, as apparently FBG concentrations already were under adequate control by the basal insulin in the BOT setting. Logically, patients in studies including insulin-naive patients had the highest baseline FBG levels and the greatest reduction in FBG over the course of the study. ${ }^{15}{ }^{16}$ However, the majority of this improvement occurred during the basal insulin run-in phase, prior to prandial insulin initiation. Consequently, in line with current guidelines, ${ }^{1}$ basalplus patients in clinical studies are typically those with well-controlled fasting glucose levels, but inadequately high postprandial excursions leading to unsatisfactorily high HbAlc.

Tight glycemic control is particularly important in hospitalized patients, given the associations between hyperglycemia/hypoglycemia and higher rates of infection, increased length of hospital stay and mortality. ${ }^{29-32}$ Therefore, insulin regimens are commonly employed in non-critical-care patients, regardless of prior diabetes management approaches. Indeed, subjects in basal-plus inpatient studies were previously managed by diet, OADs only or low-dose insulin. ${ }^{1825}$ The greatest risk of abrupt implementation of insulin therapy and tight titration in inpatients represents hypoglycemia. Accordingly, a basal-plus approach may be more appropriate than a full basal-bolus regimen in this particular population, supported by the lower rates of hypoglycemia reported by Umiperrez et al ( $5 \%$ vs $16 \%$ for basal-plus and basal-bolus patients, respectively). ${ }^{18}$ Conversely, little differences were observed between basal-plus and premixed insulin regimens in this context. ${ }^{25}$ However, the former allows for a greater degree of flexibility, which may have particular utility in a volatile hospital setting. In summary, any non-ICU hospitalized patient with suboptimal glycemic control may be a potential basal-plus candidate, although supporting literature is sparse.

A further consideration for all patients requiring insulin intensification is their individual risk of hypoglycemia. Two meta-analyses ${ }^{33} 34$ combining data from 4 of the 15 clinical studies included in the present systematic review (OPAL, ELENOR, POC and 1-2-3- trials) 89151721 identified female gender, a longer diabetes duration and higher IGlar doses to be predictive of symptomatic hypoglycemia on a basal-plus regimen. ${ }^{33} 34$ Finally, we recommend that caution and close monitoring should be employed in such patients, with alternative intensification methods such as addition of lixisenatide meriting consideration.

\section{Basal and bolus timing and approaches to titration}

Times of the day for basal insulin administration were not prespecified in most of the trials, except the studies of Raccah, Jin, Tinhones and Gross, where insulin was injected in the evening. ${ }^{1420} 22{ }^{26}$ In most of the trials, the basal insulin was not further titrated on addition of the short-acting insulin, but rather kept at stable doses. Moreover, in all studies the long-acting basal insulin analogs IGlar and IDet as opposed to neutral protamin hagedorn $(\mathrm{NPH})$ insulin were used. Therefore, daytime differences in basal insulin applications may be negligible.

Several basal-plus trials have compared the effect of administering short-acting insulin at different mealtimes. For both IGlar/IGlu and IGlar/ILis basal-plus regimens, reduction in HbAlc was found to be unaffected by the timing of short-acting insulin administration. ${ }^{8}{ }^{14}$ This suggests that preferential dosing at breakfast, lunch or dinnertime has little bearing on glycemic efficacy, and that a degree of flexibility may be acceptable. However, in the subset of patients with baseline $\mathrm{HbAlc}>7 \%$, achievement of a value below this target was more common when their preprandial IGlu bolus was administered prior to their main meal of the day. ${ }^{8}$ Consequently, administration of short-acting insulin with the patient's largest meal may be recommended. This begs the question of how to define the main meal. The STEPWISE trial assessed outcomes of the basal-plus regimen when the main meal was defined as the "largest meal from the patient's perspective" (with titrations based on preprandial blood glucose) compared with the "meal with the largest postprandial glucose excursion," and found no significant differences between these groups. ${ }^{28}$ This suggests that either method is valid, although larger-scale studies would be helpful to confirm this.

A slightly smaller degree of weight gain appears to be associated with the use of ILis before a main evening meal compared with breakfast or lunch, ${ }^{14}$ suggesting that evening administration may be more attractive to patients for whom weight is of particular concern. However, this difference appears to be of minimal clinical significance, with the magnitude of the gains shown to be small and similar to those on BOT alone. ${ }^{21}$ Indeed, considering that more hypoglycemia has also been reported in patients administering their prandial insulin in the evening, ${ }^{8}{ }^{14}$ patients may gain a greater safety benefit from consuming their main meal (and administering their short-acting insulin) at breakfast or lunchtime. 
These factors collectively need to be taken into account when establishing individual treatment strategies.

A number of factors have been used to determine the need for insulin dose titration or stepwise addition of additional prandial boluses in basal-plus patients. Basing treatment decisions on telecare monitoring or SMBG was found to result in comparable glycemic improvements, weight changes and hypoglycemia incidences, ${ }^{15}$ as was the use of preprandial or postprandial blood glucose readings. ${ }^{28}$ This suggests that any of the above may be appropriate for informing insulin requirements. Analysis of the economic value of each method alongside assessment of convenience and patient satisfaction would allow for more informed selection.

\section{Basal-plus versus basal-bolus}

The majority of studies found the basal-plus approach to be similar to the basal-bolus regimen in terms of change in $\mathrm{HbAlc}^{9}{ }^{27}$ and daily blood glucose, ${ }^{18}$ with only the OSIRIS trial failing to report non-inferiority. ${ }^{20}$ However, there appears to be a consistent trend toward fewer basalplus than basal-bolus patients meeting a $<7 \%$ HbAlc target at around 6 months, despite not always reaching significance. ${ }^{9027}$ Interestingly, the FullSTEP study found that by 32 weeks, this difference had disappeared, with comparable proportions attaining HbAlc $<7 \% .{ }^{27}$ This is in keeping with the idea that basal-plus is a more gradual approach to treatment intensification after BOT, likely requiring more time to achieve satisfactory glycemic control than the immediate intensive basal-bolus regimen. This should be taken into account when planning the duration of future basal-plus comparative studies, and also kept in mind by physicians, so that unnecessary delays do not hinder patient progress. Nevertheless, the basal-plus regimen has been shown by several studies to result in comparably less hypoglycemia (including severe hypoglycemia) ${ }^{91827}$ and weight gain ${ }^{162027}$ than basal-bolus, with fewer daily injections required. Only the OSIRIS trial failed to note a reduction in hypoglycemia, with only Davidson et al reporting no difference in weight gain. ${ }^{920}$ These are likely explained by differences in study design and study populations, such as the extent to prior insulin exposure, dosage and use of OADs. In summary, basal-plus regimens may be more acceptable to patients in need of, but resistant to, treatment intensification due to concerns over weight gain and hypoglycemia risk, as well as reluctance to multiple daily injections.

\section{Basal-plus versus premixed insulin}

Most studies comparing basal-plus and premixed insulin regimens show the former to be non-inferior for reduction in HbAlc, ${ }^{16} 2223$ with an inpatient study reporting comparable improvements in daily blood glucose. ${ }^{25}$ Furthermore, at least comparable proportions of patients are reported to achieve HbA1c $<7 \%,{ }^{16} 222326$ with hypoglycemia and weight gain also found to be similar by the majority of studies. ${ }^{1622232526}$ On balance, it appears that premixed insulin and basal-bolus approaches result in similar improvements in glycemic control, with a comparable degree of safety. Furthermore, the number of necessary injections (two per day) is generally identical in each regimen, meaning that neither has a particular advantage in this respect. However, the addition of one prandial bolus to a previously established BOT regimen may be simpler to implement and allow a greater degree of flexibility in terms of dose titrations. Thus, convenience and individual patient preference are likely to be the most relevant factors when deciding between basalplus and premixed insulin regimens.

\section{Basal-plus versus GLP-1 RA}

In recent years, the addition of a GLP-1 RA to optimized basal insulin has been explored for improving glycemic control in patients with T2DM. Although several studies have compared a basal/GLP-1 RA regimen to a basalbolus regimen, only one trial (GetGoal Duo-2) appears to have included a basal-plus arm. ${ }^{24}$ In this particular study, reductions in HbAlc were non-inferior in patients on lixisenatide compared with those on a basal-plus regimen. In accordance with the published literature,$^{35}$ however, a small amount of weight loss $(-0.6 \mathrm{~kg})$ was seen in patients on lixisenatide, while basal-plus patients experienced a small weight gain $(+1 \mathrm{~kg})$. In addition, lixisenatide was associated with lower rates of hypoglycemia, with a complete absence of severe symptomatic hypoglycemia. This suggests that the main aims of antidiabetic treatment (namely improved glycemic control without excessive weight gain or hypoglycemia) may be better met by a basal/GLP-1 RA than basal-plus regimen. However, GLP-1 RAs have been associated with gastrointestinal side effects, affecting $35.2 \%$ versus $8.6 \%$ of the lixisenatide and basal-plus patients in the GetGoal Duo-2 Trial, respectively. ${ }^{24}$ Increased heart rate ${ }^{36}$ and kidney damage ${ }^{37}$ have also been associated in some studies with GLP-1 RAs. These adverse effects may significantly impact quality of life, adherence and persistence, undermining the advantage gained from fewer insulin injections compared with the basal-bolus approach. Consequently, all the above factors should be carefully considered when individualizing patient care plans.

\section{Basal-plus insulin combinations}

In all but two of the studies, the basal insulin used was IGlar $100 \mathrm{U} / \mathrm{mL}$ rather than IDet. This may partly reflect an earlier approval, with IGlar having received European Medicines Agency/Food and Drug Administration approval several years before IDet (2000/2000 vs 2004/2005). A meta-analysis comparing these two long-acting insulins found them to be comparable for target HbAlc achievement without hypoglycemia when used as part of a BOT regimen ( $\mathrm{RR}=1.07 ; 95 \% \mathrm{CI}$ : 0.87 to 1.33), as well as risk of symptomatic hypoglycemia (RR $=0.99 ; 95 \%$ CI: 0.90 to 1.08$).{ }^{38}$ Similarly, pharmacodynamic studies have demonstrated very little difference in duration of action. ${ }^{39}$ However, IGlar has been associated with a lower rate of adverse events leading to treatment 
discontinuation $(\mathrm{RR}=0.40 \text {; } 95 \% \mathrm{CI}: 0.24 \text { to } 0.69)^{38}$ and a lower daily insulin dose requirement $(\mathrm{RR}=0.29$; $95 \% \mathrm{CI}$ : 0.25 to 0.32$).{ }^{40-42}$ The latter points toward potential cost savings and may be an additional explanation for the greater use of IGlar in the present group of studies. IDet may result in less weight gain $(0.6 \pm 2.5 \mathrm{vs} 4.2 \pm 4.1 \mathrm{~kg}$, $\mathrm{p}=0.004),{ }^{43}$ and all these factors must be considered when selecting basal insulins. Head-to-head studies comparing IGlar and IDet basal-plus regimens would be informative. The use of the newer, long-acting insulins-degludec and IGlar-300 - are outside the context of this review but warrant similar investigation.

When deciding on the best prandial insulin to add to a BOT regimen, several rapid-acting analogs (IGlu, IAsp and ILis) are available. IGlu has been shown to provide similar or superior improvements in glycemic control compared with regular human insulin at similar doses, with less severe postadministration excursions and reduced hypoglycemia. ${ }^{44}$ In the studies included in the present analysis, the most recently approved analog IGlu was most commonly used in the basal-plus arm followed by ILis and IAsp. This preference may also be a reflection of its slightly faster onset of action compared with the latter two analogs, as demonstrated by a number of comparative studies. ${ }^{46-48}$ Theoretically, this allows more flexible bolus administration at mealtime. ${ }^{49}$ It is difficult to draw evidence supporting the use of any one particular analog from the present studies due to their heterogeneity. Direct head-to-head comparisons of different basal/prandial insulin combinations in a larger population would be necessary to address this question.

\section{Limitations}

This review is based on studies identified through a PubMed search and the recommendations of experts. Other data sources, such as Embase, Cochrane and Medline, may have identified other studies that could have been included in this manuscript. There is a high degree of heterogeneity between the included studies in terms of design (including aims, sample sizes, durations, inpatient vs outpatient settings, definitions of hypoglycemia and insulin combinations) and baseline patient characteristics (age, glycemic levels, use of insulin and OADs). Furthermore, the definition of basal-plus is variable, with some studies referring to the addition of just one prandial bolus per day, others to a stepwise addition of up to three boluses based on target achievement over time and some to the administration of short-acting insulin only where prandial blood glucose readings indicate its necessity. This begs for standardization of terms used in the field of diabetes to avoid confusion in future. This heterogeneity contributed complexity to a qualitative evaluation and precluded the performance of a meta-analysis. Furthermore, most of the included studies were RCTs. While this suggests high-quality data and validity of interstudy comparisons, tight inclusion criteria mean that study samples represent selected populations, limiting their generalizability to the overall population in daily clinical practice. Of note, a lack of blinding in most of the trials also means that the effect of experimenter bias cannot be ruled out.

\section{CONCLUSIONS}

In outpatients with T2DM, the addition of a single shortacting insulin bolus to a pre-existing BOT regimen results in efficient reductions in $\mathrm{HbAlc}$, with minimal weight gain and low rates of hypoglycemia. There is also separate evidence for its safe and effective use in an inpatient setting. While basal-plus and premixed insulin regimens appear to result in similar outcomes, a greater degree of flexibility is possible with the former approach. Furthermore, basal-plus has been associated with less weight gain, hypoglycemia and injection requirements compared with basal-bolus, with similar HbAlc effects. The translation of these proven advantages into less resistance to insulin intensification and reduction of clinical inertia remains to be demonstrated. Interestingly, the addition of lixisenatide and liraglutide to basal insulin instead of prandial insulin may be advantageous in terms of weight and hypoglycemia, but gastrointestinal side effects remain a drawback. Thus, multiple factors must be considered when formulating treatment plans. The availability of different insulin combinations for use in a basal-plus regimen is advantageous in terms of clinical flexibility, but comparative studies to identify patients most likely to benefit from each particular pairing would be informative.

Acknowledgements We acknowledge the help of Helen Sims (Institute for Pharmacology and Preventive Medicine) with the compilation of the evidence and for editing the manuscript.

Contributors PB and JS drafted the first version of the manuscript and all authors revised the article for important intellectual content. All authors approved the final version for publication.

Funding Sanofi Aventis Deutschland GmbH supported the systematic literature search underlying this article.

Competing interests JS has attended Speaker's Bureaux for AstraZeneca, BristolMyers Squibb, Bayer Healthcare, Berlin Chemie, Boehringer Ingelheim, Eli Lilly, GlaxoSmithKline, Lifescan, Merck Sharp \& Dohme, Mundipharma, Novartis, Novo Nordisk, Pfizer, Sanofi and Takeda. PB has received research funding and honoraria for consultancy from AstraZeneca, Bristol-Myers Squibb, Novartis and Sanofi. AB is an employee of Sanofi.

Patient consent for publication Not required.

Provenance and peer review Not commissioned; externally peer reviewed.

Open access This is an open access article distributed in accordance with the Creative Commons Attribution Non Commercial (CC BY-NC 4.0) license, which permits others to distribute, remix, adapt, build upon this work non-commercially, and license their derivative works on different terms, provided the original work is properly cited, appropriate credit is given, any changes made indicated, and the use is non-commercial. See: http://creativecommons.org/licenses/by-nc/4.0/.

\section{ORCID iD}

Peter Bramlage http://orcid.org/0000-0003-4970-2110

\section{REFERENCES}

1. Davies MJ, D'Alessio DA, Fradkin J, et al. Management of hyperglycemia in type 2 diabetes, 2018. A consensus report by the American diabetes association (ADA) and the European association for the study of diabetes (EASD). Diabetes Care 2018;41:2669-701.

2. Monnier L, Colette C, Owens D. Postprandial and basal glucose in type 2 diabetes: assessment and respective impacts. Diabetes Technol Ther 2011;13(Suppl 1):S25-32. 
3. Brod M, Kongsø JH, Lessard S, et al. Psychological insulin resistance: patient beliefs and implications for diabetes management. Quality of Life Research 2009;18.

4. Kunt T, Snoek FJ. Barriers to insulin initiation and intensification and how to overcome them. Int J Clin Pract 2009;63(1 Suppl.):6-10.

5. Khunti K, Millar-Jones D. Clinical inertia to insulin initiation and intensification in the UK: a focused literature review. Prim Care Diabetes 2017;11:3-12.

6. Rizvi AA. Treatment of type 2 diabetes with biphasic insulin analogues. Eur Med J Diabetes 2016;4:74-83.

7. Rizvi AA, Ligthelm RJ. The use of premixed insulin analogues in the treatment of patients with type 2 diabetes mellitus: advantages and limitations. Insulin 2007;2:68-79.

8. Lankisch MR, Ferlinz KC, Leahy JL, et al. Introducing a simplified approach to insulin therapy in type 2 diabetes: a comparison of two single-dose regimens of insulin glulisine plus insulin glargine and oral antidiabetic drugs. Diabetes Obes Metab 2008;10:1178-85.

9. Davidson M, Raskin P, Tanenberg R, et al. A stepwise approach to insulin therapy in patients with type 2 diabetes mellitus and basal insulin treatment failure. Endocrine Practice 2011;17:395-403.

10. Owens DR, Schalkwyk Cvan, Smith P, et al. Algorithm for the introduction of rapid-acting insulin analogues in patients with type 2 diabetes on basal insulin therapy. Pract Diab Int 2009;26:70-7.

11. Liberati A, Altman DG, Tetzlaff J, et al. The PRISMA statement for reporting systematic reviews and meta-analyses of studies that evaluate healthcare interventions: explanation and elaboration. $B M J$ 2009;339:b2700.

12. Schardt C, Adams MB, Owens T, et al. Utilization of the PICO framework to improve searching PubMed for clinical questions. BMC Med Inform Decis Mak 2007;7:16.

13. Choe EY, Lee Y-ho, Lee B-W, et al. Glycemic effects of once-a-day rapid-acting insulin analogue addition on a basal insulin analogue in Korean subjects with poorly controlled type 2 diabetes mellitus. Diabetes Metab J 2012;36:230-6.

14. Gross JL, Rojas A, Shah S, et al. Efficacy and safety of a premixed versus a basal-plus insulin regimen as intensification for type 2 diabetes by timing of the main meal. Curr Med Res Opin 2016;32:1109-16.

15. Prato SD, Nicolucci A, Lovagnini-Scher AC, et al. Telecare provides comparable efficacy to conventional Self-Monitored blood glucose in patients with type 2 diabetes titrating one injection of insulin Glulisine-the ELEONOR study. Diabetes Technol Ther 2012:14:175-82.

16. Riddle MC, Rosenstock J, Vlajnic A, et al. Randomized, 1-year comparison of three ways to initiate and advance insulin for type 2 diabetes: twice-daily premixed insulin versus basal insulin with either basal-plus one prandial insulin or basal-bolus up to three prandial injections. Diabetes Obes Metab 2014;16:396-402.

17. Nicolucci A, Del Prato S, Vespasiani G, et al. Optimizing insulin glargine plus one injection of insulin glulisine in type 2 diabetes in the ELEONOR study: similar effects of telecare and conventional self-monitoring of blood glucose on patient functional health status and treatment satisfaction. Diabetes Care 2011;34:2524-6.

18. Umpierrez GE, Smiley D, Hermayer K, et al. Randomized study comparing a Basal-bolus with a basal plus correction insulin regimen for the hospital management of medical and surgical patients with type 2 diabetes: basal plus trial. Diabetes Care 2013;36:2169-74

19. Haw JS, Farrokhi F, Smiley D, et al. Comparison of basal insulin regimens on glycemic variability in NONCRITICALLY ill patients with type 2 diabetes. Endocrine Practice 2015;21:1333-43.

20. Raccah $D$, Haak TJ, Huet $D$, et al. Comparison of stepwise addition of prandial insulin to a basal-bolus regimen when basal insulin is insufficient for glycaemic control in type 2 diabetes: results of the Osiris study. Diabetes Metab 2012;38:507-14.

21. Owens DR, Luzio SD, Sert-Langeron C, et al. Effects of initiation and titration of a single pre-prandial dose of insulin glulisine while continuing titrated insulin glargine in type 2 diabetes: a 6-month 'proof-of-concept' study. Diabetes Obes Metab 2011;13:1020-7.

22. Jin S-M, Kim JH, Min KW, et al. Basal-prandial versus premixed insulin in patients with type 2 diabetes requiring insulin intensification after basal insulin optimization: a 24-week randomized noninferiority trial. J Diabetes 2016;8:405-13.

23. Vora J, Cohen N, Evans M, et al. Intensifying insulin regimen after basal insulin optimization in adults with type 2 diabetes: a 24-week, randomized, open-label trial comparing insulin glargine plus insulin glulisine with biphasic insulin aspart (LanScape). Diabetes Obes Metab 2015;17:1133-41.

24. Rosenstock J, Guerci B, Hanefeld M, et al. Prandial options to advance basal insulin glargine therapy: testing Lixisenatide plus basal insulin versus insulin Glulisine either as Basal-Plus or Basal-
Bolus in type 2 diabetes: the GetGoal Duo-2 trial. Diabetes Care 2016;39:1318-28.

25. Gracia-Ramos AE, Cruz-Domínguez MdelP, Madrigal-Santillán $\mathrm{EO}$, et al. Premixed insulin analogue compared with Basal-Plus regimen for inpatient glycemic control. Diabetes Technol Ther 2016;18:705-12.

26. Tinahones FJ, Gross JL, Onaca A, et al. Insulin lispro low mixture twice daily versus basal insulin glargine once daily and prandial insulin lispro once daily in patients with type 2 diabetes requiring insulin intensification: a randomized phase IV trial. Diabetes Obes Metab 2014;16:963-70.

27. Rodbard HW, Visco VE, Andersen $\mathrm{H}$, et al. Treatment intensification with stepwise addition of prandial insulin aspart boluses compared with full basal-bolus therapy (FullSTEP study): a randomised, treat-to-target clinical trial. Lancet Diabetes Endocrinol 2014;2:30-7.

28. Meneghini L, Mersebach H, Kumar S, et al. Comparison of 2 intensification regimens with rapid-acting insulin aspart in type 2 diabetes mellitus inadequately controlled by once-daily insulin detemir and oral antidiabetes drugs: the step-wise randomized study. Endocr Pract 2011;17:727-36.

29. Pomposelli JJ, Baxter JK, Babineau TJ, et al. Early postoperative glucose control predicts nosocomial infection rate in diabetic patients. JPEN J Parenter Enteral Nutr 1998;22:77-81.

30. Bruno A, Gregori D, Caropreso A, et al. Normal glucose values are associated with a lower risk of mortality in hospitalized patients. Diabetes Care 2008;31:2209-10.

31. Noordzij PG, Boersma E, Schreiner F, et al. Increased preoperative glucose levels are associated with perioperative mortality in patients undergoing noncardiac, nonvascular surgery. Eur J Endocrinol 2007:156:137-42.

32. McAlister FA, Majumdar SR, Blitz S, et al. The relation between hyperglycemia and outcomes in 2,471 patients admitted to the hospital with community-acquired pneumonia. Diabetes Care 2005;28:810-5.

33. Seufert J, Brath H, Pscherer S, et al. Composite efficacy parameters and predictors of hypoglycaemia in basal-plus insulin therapy-a combined analysis of 713 type 2 diabetic patients. Diabetes Obes Metab 2014:16:248-54.

34. Lankisch MR, Del Prato S, Dain M-P, et al. Use of a basal-plus insulin regimen in persons with type 2 diabetes stratified by age and body mass index: a pooled analysis of four clinical trials. Prim Care Diabetes 2016;10:51-9.

35. Carris NW, Taylor JR, Gums JG. Combining a GLP-1 receptor agonist and basal insulin: study evidence and practical considerations. Drugs 2014;74:2141-52.

36. Russell-Jones D, Cuddihy RM, Hanefeld M, et al. Efficacy and safety of exenatide once Weekly versus metformin, pioglitazone, and sitagliptin used as monotherapy in drug-naive patients with type 2 diabetes (DURATION-4): a 26-week double-blind study. Diabetes Care 2012;35:252-8.

37. Weise WJ, Sivanandy MS, Block CA, et al. Exenatide-associated ischemic renal failure. Diabetes Care 2009;32:e22-3.

38. Rys P, Wojciechowski P, Rogoz-Sitek A, et al. Systematic review and meta-analysis of randomized clinical trials comparing efficacy and safety outcomes of insulin glargine with NPH insulin, premixed insulin preparations or with insulin detemir in type 2 diabetes mellitus. Acta Diabetol 2015;52:649-62.

39. Luzio SD, Dunseath GJ, Atkinson MD, et al. A comparison of the pharmacodynamic profiles of insulin detemir and insulin glargine: a single dose clamp study in people with type 2 diabetes. Diabetes Metab 2013;39:537-42.

40. Zhuang YG, Peng H, Huang F. A meta-analysis of clinical therapeutic effect of insulin glargine and insulin detemir for patients with type 2 diabetes mellitus. Eur Rev Med Pharmacol Sci 2013;17:2566-70.

41. Swinnen SGHA, DeVries JH. Higher dose requirements with insulin detemir in type 2 diabetes-three cases and a review of the literature. Diabetes Res Clin Pract 2009;84:e24-6.

42. Guillermin A-L, Samyshkin Y, Wright D, et al. Modeling the lifetime costs of insulin glargine and insulin detemir in type 1 and type 2 diabetes patients in Canada: a meta-analysis and a costminimization analysis. J Med Econ 2011;14:207-16.

43. Elisha B, Azar M, Taleb N, et al. Body composition and epicardial fat in type 2 diabetes patients following insulin detemir versus insulin glargine initiation. Horm Metab Res 2016;48:42-7.

44. Garg S, Ampudia-Blasco F, Pfohl M. Rapid-acting insulin analogues in Basal-bolus regimens in type 1 diabetes mellitus. Endocr Pract 2010;16:486-505.

45. Home PD. The pharmacokinetics and pharmacodynamics of rapidacting insulin analogues and their clinical consequences. Diabetes Obes Metab 2012;14:780-8. 
46. Heise T, Nosek L, Spitzer $\mathrm{H}$, et al. Insulin glulisine: a faster onset of action compared with insulin lispro. Diabetes Obes Metab 2007;9:746-53.

47. Luzio S, Peter R, Dunseath GJ, et al. A comparison of preprandial insulin glulisine versus insulin lispro in people with type 2 diabetes over a 12-h period. Diabetes Res Clin Pract 2008;79:269-75.
48. Arnolds S, Rave K, Hövelmann U, et al. Insulin glulisine has a faster onset of action compared with insulin aspart in healthy volunteers. Exp Clin Endocrinol Diabetes 2010;118:662-4.

49. Helms KL, Kelley KW. Insulin glulisine: an evaluation of its pharmacodynamic properties and clinical application. Ann Pharmacother 2009;43:658-68. 